\title{
Verification of Malus's Law using a LCD monitor and Digital Photography
}

\author{
Verificação da Lei de Malus usando monitor LCD e fotografia digital
}

\author{
Larissa Vertchenko*1, Lev Vertchenko² \\ ${ }^{1}$ Departamento de Física, Universidade Federal de Minas Gerais, Belo Horizonte, MG, Brazil. \\ ${ }^{2}$ Departamento de Física e Química, Pontifícia Universidade Católica de Minas Gerais, Belo Horizonte, MG, Brazil.
}

Recebido em 4 de fevereiro de 2016. Aceito em 26 de abril de 2016

\begin{abstract}
An alternative laboratory experiment for verifying Malus's Law, as well as understanding concepts of photometry, polarization and image formation on liquid crystal displays using low cost materials is proposed. The experimental setup could be introduced to any undergraduate curriculum using readily available tools, such as a digital camera, polarizer removed from a cell phone and a LCD laptop screen. A fairly amount of photometric data was collected and analyzed by an astronomical image processing software resulting in a successful verification of Malus's Law.
\end{abstract}

Keywords: Polarization, Photometry, Malus's Law, Liquid Crystal, image processing software.

Propomos um experimento alternativo para verificação da Lei de Malus, assim como para o entendimento dos conceitos de fotometria, polarização e formação de imagem em displays de cristal liquido, usando materiais de baixo custo. O arranjo experimental pode ser introduzido em qualquer grade curricular dos cursos de graduação em física, utilizando ferramentas facilmente acessíveis, como a câmera fotográfica digital, um polarizador removido de um aparelho celular e um monitor LCD de um computador. Uma razoável quantidade de dados fotométricos foi coletada e analisada através de um software de processamento de imagens, verificando com sucesso a Lei de Malus.

Palavras-chave: Polarização, Fotometria, Lei de Malus, Cristal Liquido, Software de Processamento de Imagem.

\section{Introduction}

In the 19th century, French physicist Etienne Louis Malus developed research [1] about the relations between the incident angle and the reaction of light beams on polished surfaces, initiating the study of the polarization of light. Currently, polarization has a great importance in different areas of knowledge such as, for example, physics, engineering and astronomy. When students learn about polarization, they often face equipment whose operation they do not usually understand, like polarized filters and LCD screens, mostly found in electronic devices such as computers, cell phones and television sets. As a result they are not able to correlate the concept of

*Endereço de correspondência: vertlar25@gmail.com. polarization with the technology present in everyday life.

Usually, the polarization approach in didactic laboratories $[2,3]$ begins with the study of Malus's Law using linear polarizers, laser sources and a photometer [4]. However, these materials only provide a strictly quantitative experiment since the student will be just looking for the values of the intensity of light, provided by the photometer, as a function of the angle of the polarizer placed in front of the laser source. The addition of instruments belonging to the day-to-day life of the students can be very meaningful for their learning in undergraduate optics labs. In this paper, we propose a low cost experiment for verifying Malus's Law using a computer monitor, a polarizer extracted from a cell phone, a digital cam- 
era and astronomical software for image treatment. All these instruments are easily found in the backpack of any student which allows them to actively interact with their experimental apparatus instead of just passively absorb the material placed on the laboratory's table. Furthermore, the use of materials and equipment directly connected to contemporary gadgets such as computers, digital cameras or cell phones stimulate the students to explore and learn about these technologies, turning such experiments into valuable educational tools. For example, the setup here proposed requires the students to understand how a digital camera functions and discuss features available in it, like exposure time, aperture, focusing and sensitivity. Or, by extracting the components of cell phones, one can learn the concepts behind the liquid crystal display and the polarizer.

For data analysis we use graphing software and a photometric measure tool contained in the astronomical image processing software called IRIS [5]. The application of this software combined with a digital camera also permits to discuss astronomical photometry techniques and Charge Coupled Device (CCD) imaging photometry. This new technology is useful to make photometric observations even with faint objects. Nowadays, in budget price digital cameras, CCDs have been substituted by another image sensor called Complementary Metal Oxide Semiconductor (CMOS) as a result of its low cost, high noise immunity and low power consumption. However, for our purposes, the CMOS sensor works as well as a CCD sensor.

Other works $[6,7]$ involving experiments with polarization of light and LCD screens have been developed showing that students can become familiar with basic principles of physics, and more accurately, concepts of optics. In 1950, an experiment on Malus's Law [8] was also performed in an elementary laboratory with an inexpensive apparatus consisting of a photoelectric cell attached to a galvanometer. This paper intends to present an application of photometry through an alternative method to study polarization and verify Malus's Law using contemporary materials that are easy to obtain.

The rest of this paper is organized as follows. In Sec. 2 we discuss the concepts of photometry, polarization and Malus's Law. Section 3 describes the experimental realization and results. Finally, in Sec. 4 we present the conclusions about the work.

\section{Photometry, Polarization and Malus's Law}

Photometry and polarization have vast applications in many fields. In fact, their combination is behind most of consumer electronics products and accessories, such as cell phones, computers, televisions, etc. Here, we combine photometric measurements and polarization properties to verify Malus's Law with readily available tools, designing an experiment easily reproducible in any undergraduate laboratory and even at home.

\subsection{Photometry}

In optics, photometry [9] is the stimulation of visual sensation by electromagnetic radiation in a simulation of the human vision. However, due to the subjectivity of the human visual system it cannot be quantifiable in absolute physical units. Early sources used the intensity of a candle as the standard, hence the unity candela, and observers would compare an unknown light source to it. Presently, there is a standardized weight function based on the sensitivity of the human eye to a particular wavelength [10]. Applications of photometry include color and light intensity analysis, digital media, automotive industry and aerospace lighting to name a few. In sciences, photometry has been particularly important to study celestial objects.

The so-called Astronomical photometry [11] measures the radiation flux, $f_{\lambda}$, that we receive from celestial objects over broad wavelengths bands of radiation. Such measurements, usually coupled to distance estimation, can give us information on the object's luminosity, temperature, size and other properties. The luminous energy captured by a time interval $\Delta t$ over an area $A$ is given by

$$
E=f_{\lambda} A \Delta t=N h \frac{c}{\lambda}
$$

where $N$ is the number of incident photons, $\lambda$ the wavelength, $h$ Planck's constant and $c$ is the speed of light in vacuum. The term $h c / \lambda$ corresponds to the energy of each photon as proposed initially by Planck [12].

In astronomical photometry, the selection of the wavelength (or color) is defined by the optical filter used, and the flux measured by a time interval of wavelengths is obtained by the convolution of the incident flux with the transmission function of the 
filter and spectral sensibility of the CCD. However, the image formation in electronic devices, such as digital cameras and LCD monitors, use the RGB system (red, green, blue) [13], where the colors result from the combination of beams from the primary colors red, green and blue. Nevertheless, the association of these primary colors to the wavelengths is not completely defined due to its dependence of the device. The zero value corresponds to the absence of light of the pixel, while the value 255 usually corresponds to the maximum of intensity of a primary color.

The number of electrons counted on a CCD sensor, $N_{e}$, can be related to the number of incident photons $N$ by an efficiency factor $\eta$, where $N_{e}=N \eta$. Knowing this, the equation (1) can be written as

$$
N_{e}=\frac{\eta A \lambda f_{\lambda} \Delta t}{h c}
$$

showing that the number of electrons that react to light and counted on the CCD pixels is proportional to the incident flux when a time interval $\Delta t$ is held constant. This permits to convert the number of electrons that achieve the CCD in terms of luminous flux unity, such as Analog Digital Units (ADU) [14].

The delicacy of astronomical measurements and their worldwide diffusion have prompted the development of very user friendly free software for photometry. In this work, we used the IRIS astronomical photometric software whose basic features are described later on this paper.

\subsection{Polarization, Liquid Crystal Displays and Malus's Law}

Polarization is a property of electromagnetic waves related to the orientation of the oscillatory electric field vector. For example, if the electric field oscillates in a specific direction, we say that the wave is linearly polarized. This will be the situation analyzed in this paper. The alternative, when the electric field changes direction during the propagation, will generate circularly or, more generally, elliptically polarized light [15]. Not all light is polarized. In fact, the most common situation in natural sources is to observe partially polarized light corresponding to statistical mixtures of well-defined linearly independent polarizations [16]. Sometimes, it may be even impossible to define the polarization of a propagating light field but those cases are out of the scope of this text. Polarizers work as optical filters that allow the passage of light of a specific polarization. For example, linear polarizers are optical devices defined by a transmission axis, i.e. they allow the transmission of light whose electric field oscillates in a plane parallel to one particular chosen axis, and block the component of the electric field that oscillates in the orthogonal direction. Typical polarizers for visible light are made of materials such as plastic and glass.

In 1808, Malus discovered that natural light becomes polarized by reflection and that the reflected light intensity varies from a maximum to a minimum when passing through a rotating calcite crystal. Nowadays it is known that not only calcite crystals but any other linear polarizer gives the same results working as an analyzer, in other words, a linear polarizer is a type of filter which modifies a beam of light whose resulting electric vector is oscillating in one plane [17]. An analyzer consists of another linear polarizer that will allow only the parallel component of the incident wave to pass through it. Knowing that, Malus expressed this dependence between the intensity of the reflected light with the angle of the analyzer in the form

$$
I=I_{M A X} \cos ^{2} \theta
$$

where $I$ is the intensity of the transmitted light, $I_{M A X}$ the maximum intensity of the light source and $\theta$ the angle of rotation of the analyzer. This equation expresses Malus's Law. As we can see, if the incident polarized light is perpendicular to the analyzer axis $(\theta=\pi / 2)$, no light will pass through it.

Liquid Crystal Displays (LCDs) [18] also work as optical filters controlling the passage of polarized light through them and are now found in many of electronic devices, for instance, computer monitors, cell phones, wristwatches and digital cameras. The liquid crystal is a molecular substance that maintains a sort of crystalline order, though it has liquid properties. These molecules are aligned as a result of their intermolecular forces, forming long parallel chains with an orientation order. By applying an electric field to the liquid crystal, its molecules tend to modify their orientation according to the field direction. When a white unpolarized light reaches the liquid crystal some of its electric field components are blocked and it turns into polarized light. A scheme of a device that utilizes LCD technology for image displays is shown in Figure 1. The unpolar- 


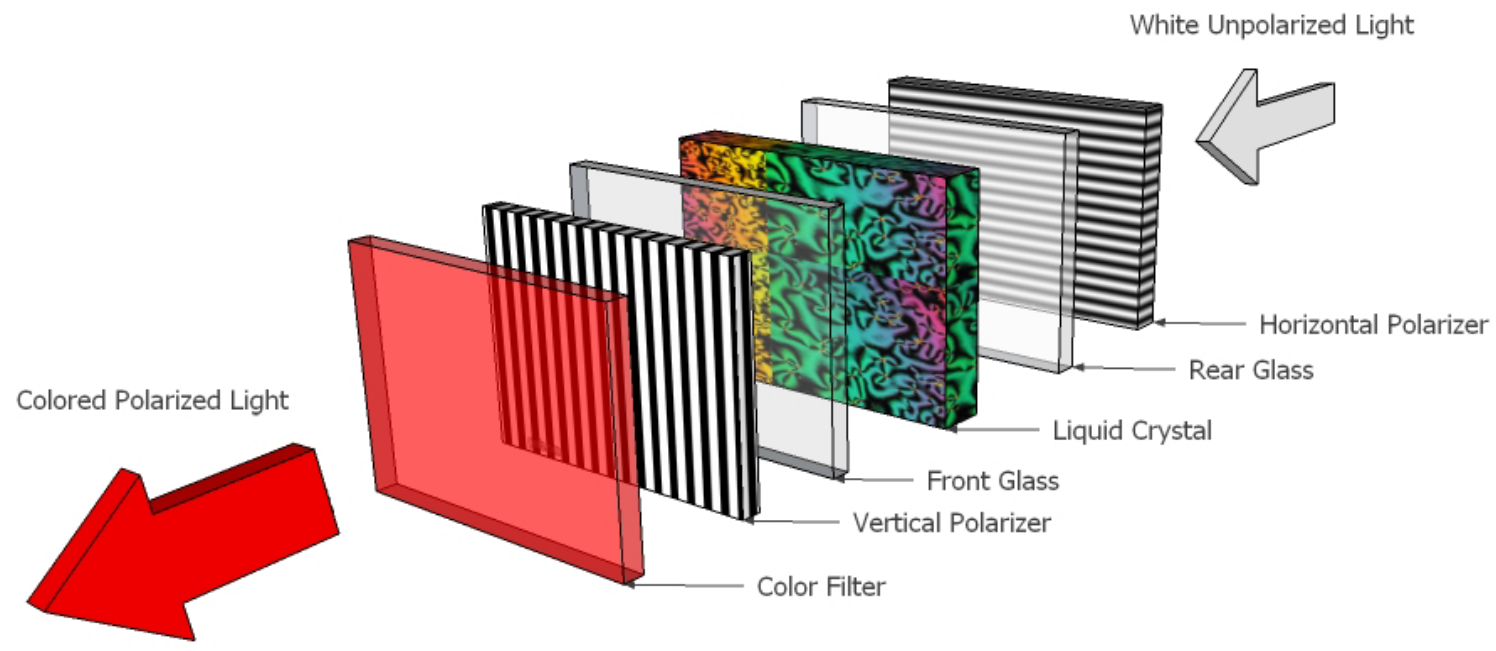

Figure 1: Schematic view of an image formation device that utilizes LCD technology. Before entering the liquid crystal the white light is horizontally polarized. Its polarization can then be rotated depending on the voltage applied across the pixel. Different voltages will correspond to different orientations of the molecules in the pixel and, concatenated with the vertical polarizer, will control the intensity of the transmitted light.

ized light passes through a polarizer called director before entering the liquid crystal, and then a second polarizer with an axis orthogonal to the first one, called analyzer, is placed after it.

\section{Experimental realization}

\subsection{Digital camera calibration}

Every photometric measurement begins with the calibration of the detector, given that photometry is based on comparing intensities of detected light. If the luminous source emits photons at a constant rate, the luminous flux becomes stationary and equation (2) also shows that the reading of the number of electrons in the CCD must keep a linear relation with the time interval $\Delta t$. However, as the efficiency factor $\eta$ is not necessarily constant, it can cause some deviation from the linear regime.

In this section, we present the methodology applied in a previous work [19] for the calibration of a digital camera for photometric measurements. We intend to, after we have selected a fixed color (wavelength) and a maximum value for the intensity of light, obtain a linear response to variation of brightness from our detector, the digital camera, when the exposure time is maintained constant, which is regulated by the shutter speed. This linear response is permitted due to the constancy of the efficiency factor $\eta$. One possible way to infer this factor is to maintain the intensity of the source constant and vary the exposure time.

To ensure that the digital camera had a linear response to the change of intensity of light, we had to perform a calibration using its manual mode. At this point it is essential to understand all the functions of a digital camera in order to manually choose the values of the parameters required for a proper light detection. We observed that in automatic mode the camera adjusts to the environment's luminosity, so when we rotated the polarizer placed in front of a luminous disc drawn on the laptop screen changing the disc's intensity the camera would also change its parameters. The calibration setup consists of a digital camera aligned to a luminous disc drawn on a laptop screen. The disc will constitute our source and we have arbitrarily chosen the red color (represented by the code $(255,0,0)$ in the RGB system) . It is important to put the screen luminosity of the laptop on its minimum, so it won't interfere in the intensity measurements.

In order to have clear images observed on the laptop screen it was necessary to adjust the following parameters [20]: sensitivity (ISO), shutter speed (S), aperture (f-number) and exposure value (EV). ISO measures the sensitivity of the image sensor inside the digital camera. The higher the ISO number, the more sensitive the sensor is and therefore brighter is the image. The shutter speed influences the time to expose light into the camera sensor and when combined with motion of objects, can make images 
blurred or clear. If a sensor has a linear response to a luminous stimulus, the energy detected must be proportional to exposure time, and, therefore, to the inverse of shutter speed. The aperture determines how much light passes to the camera sensor and the EV combines the shutter speed and f-number. It is important that these parameters do not fluctuate during the realization of the experiment.

At first, we settled a fixed sensitivity and took five pictures, using the digital camera SONY CYBERSHOT HX1 in manual mode, for different shutter speed values. The next step was to measure the intensity of each disc using the image processing software IRIS. It is also possible to perform the measurements with other softwares, but we chose this one due to its practicality and simple template. This software permits students to load the pictures taken and use the function of photometry analysis to get intensity values. Note, however, that it is important to select an appropriate radius of the boundary circle for the intensity count so that the image of the disc is completely inside the selected area as shown in Figure 2. The aperture photometry tool can be employed to conduct high precision measurements and its intensity values are given in Analog Digital Unit (ADU).

For a red luminous disc, three measures for different values of sensitivity $(\mathrm{ISO}=125, \mathrm{ISO}=400$,
ISO $=800$ ), while varying the shutter speed, were performed. In Figure 3 we present results of the intensity normalized by its maximum value as a function of the shutter speed, which is normalized by its minimum value, corresponding to the maximum of the exposure time. Plots were made using the ORIGIN [21] graphing utility. It is important to note that we chose the color of the luminous

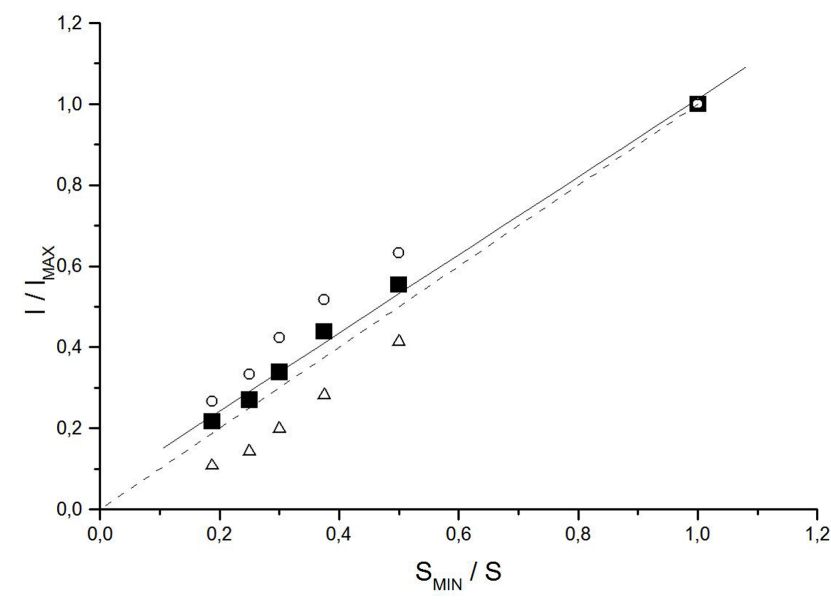

Figure 3: Plot of the relation $\mathrm{I} / \mathrm{I}_{\mathrm{MAX}}$ as a function of $\mathrm{S}_{\mathrm{MIN}} / \mathrm{S}$. The dashed line represents the identity function and the linear fit of ISO 400 (squares) is represented by the solid line. We can observe a small saturation effect for ISO 800 (circles), and a deficit of sensitivity for ISO 125 (triangles).

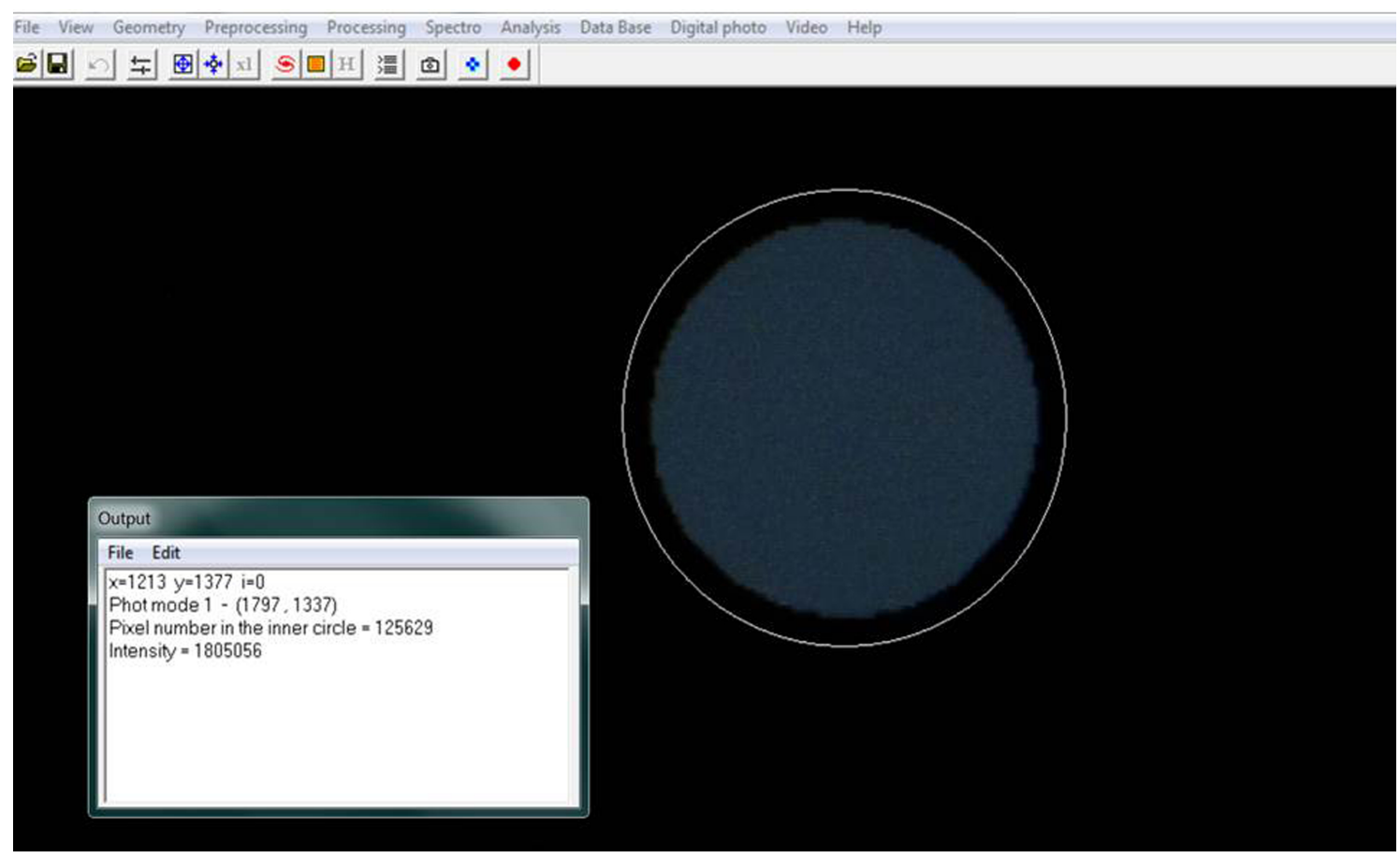

Figure 2: Picture of IRIS software showing the boundary circle and the value of the disc intensity. 
disc randomly. If another color is chosen, the same procedures for calibration must be taken in order to obtain a linear relation between the measured intensity flux and exposure time.

From the graphic we can observe that the data relative to the extreme angles coincide with the ones of a reference curve given by the identity function. Nevertheless, for the intermediate angles, the experiment shows a small deviation from the reference curve. This experimental situation can be explained by a deviation from proportionality of the light intensity registered by the CMOS sensor of the digital camera, which was measured by photometry, and the incident light intensity. To be more specific, a low light sensitivity gives a curve that is concave upward, due to the lack of sensor response for low intensities; meanwhile the saturation of the sensor gives a curve that is concave downward due to the low variation for high intensities. Therefore we can conclude that the data for the ISO $=800$ shows a small saturation effect while the red disc's data for ISO $=125$ shows a small deficit of sensitivity. For ISO 400 the linear fit gives a linear coefficient of A $=(0.00 \pm 0.02)$ and inclination $\mathrm{B}=(1.11 \pm 0.05)$, which is close to the expected values $\mathrm{A}=0$ and $\mathrm{B}$ $=1$, so this ISO value will correspond to a standard parameter for future measurements. Once the photographic apparatus is calibrated to work in the linear response regime, the radiation flux detected will be proportional to the incident radiation if we maintain the exposure time constant.

\subsection{Experimental setup and procedures for verification of Malus's Law}

We started the experimental setup to verify Malus's Law (shown in Figure 4.) by removing the LCD and polarizers from a used cell phone and linking them to a swivel base coupled to a protractor. This montage will represent the analyzer system. When looking at the parts of the cell phone it is important to see that polarizers are already bound to the liquid crystal. However, it was not necessary to separate them since there was no electric field applied to the liquid crystal that, therefore, did not interfere with the experimental results. A colored disc drawn on the black background of the laptop screen is used as the light source. Any simple program such as Microsoft Paint [22] can be used to generate the disc. It is also necessary to calibrate the analyzer system by matching the angles of the polarizer with those of the protractor. To do that, we need the angle considered as 90 degrees to be the one corresponding to the minimum of the transmitted intensity.

The next step was to align the digital camera with the polarizer and the luminous disc as illustrated in Figure 4. All the measures were performed using the digital camera parameters when it was being calibrated. Data analyzing was made using the image processing software IRIS and plots were made using ORIGIN.

In our setup we aligned the light source with the analyzer system and the digital camera as shown in Figure 4. Two sets of data were acquired for each

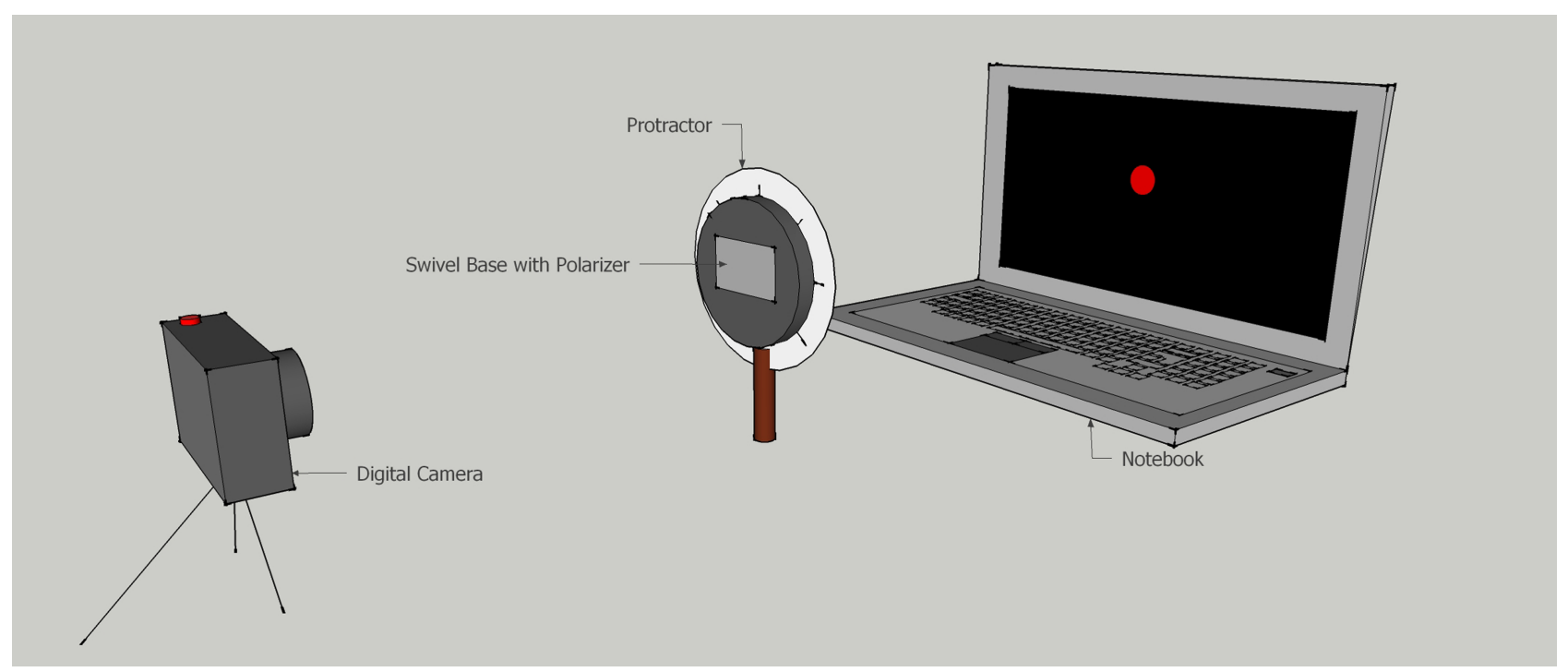

Figure 4: A scheme of the experimental setup showing the light source aligned with the analyzer system and the digital camera. 
experiment: one using a commercial linear polarizer and another using a polarizer removed from a cell phone. All pictures were taken using the digital camera SONY CYBERSHOT HX1 in manual mode. The measurements were performed by taking five pictures of the luminous disc for each angle of the analyzer, ranging from 0 to 90 degrees. The repetition of five pictures per angle allows the calculation of an average of intensity reducing the error in each measure. As an example we took five pictures for every fifteen degrees starting with the $0^{\circ}$ mark.

A total of 70 measurements were performed and the determination of the intensity of the disc in each picture was made by IRIS software. The values of the intensity given by IRIS were then used to obtain the average intensity per angle together with its standard deviation. From the data tabulated, the values of $I / I_{M A X}$ were computed as a function of the values of $\cos ^{2} \theta$ and two plots (one for each polarizer) were created using the software ORIGIN. All the measured curves have been linearly fitted and compared with the relation between intensity and angles described by Malus's Law.

\subsection{Results analysis}

For the polarizer removed from a cell phone, the digital camera parameters used were: ISO 400, S 15, f 6.3 and EV -2.0. After performing the linear fit to the data, shown in Fig. 5, we obtained the linear coefficient $\mathrm{A}=(-0.03 \pm 0.02)$ and the angular coef-

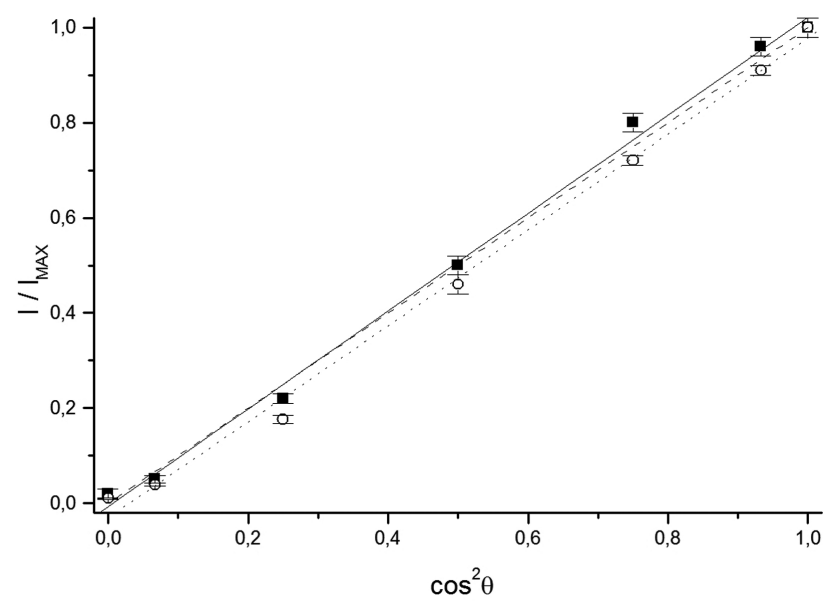

Figure 5: Plot of the relation $\mathrm{I} / \mathrm{I}_{\mathrm{MAX}}$ as a function of $\cos ^{2} \theta$ for a linear polarizer removed from a cell phone (circles) and commercial linear polarizer (squares) with their respective error bars and linear fits. The solid line represents the relation predicted by Malus's Law. ficient $\mathrm{B}=(1.01 \pm 0.03)$. The correlation coefficient was $\mathrm{R}=0.9978$.

From the graph we can observe that the data relative to extreme angles coincide exactly with the ones predicted by Malus's Law, as for $\theta=90^{\circ}$ we have no light passing through the analyzer, whereas for $\theta=0^{\circ}$ we have a normalized maximum of the intensity. Nevertheless, for the intermediate angles, the experiment shows a slight deviation from what was expected by Malus's Law. This deviation can be explained by the fact that the polarizer removed from a cell phone was attached to glass, which worked as a mirror lowering the transmittance of the polarizer. This situation can be compared to the low light sensitivity shown in Figure 3.

For the commercial linear polarizer, the digital camera parameters used were: ISO 400, S 15, f 6.3 and EV -2.0. After performing the linear fit we obtained the following values for the linear and angular coefficients: $\mathrm{A}=(-0.01 \pm 0.01)$ and $\mathrm{B}=$ $(1.02 \pm 0.03)$, showing compatible values with the ones predicted by Malus's Law which are $\mathrm{A}=0$ (no residual intensity) and $\mathrm{B}=1\left(\mathrm{I} / \mathrm{I}_{M A X}=\cos ^{2} \theta\right)$. The correlation coefficient was $\mathrm{R}=0.997$. Therefore we can conclude that the commercial linear polarizer and the one removed from a cell phone can both be used to verify Malus's Law with satisfactory results.

\section{Conclusions}

We have proposed a low cost experiment that can be performed in most laboratories using materials that are close to students. The experiment allows introducing concepts of photometry, polarization and LCD technology. Although digital cameras of the popular use are not destined for photometric measurements, we show that with adequate calibration it is possible to do so. After achieving a linear relation between the intensity flux and the exposure time we were able to successfully verify the Malus's Law. The results permit to attest the properties of light emitted from an LCD monitor and the behavior of polarizers removed from a cell phone.

We believe that in this way, both the concept of polarization and its role in the image formation on LCD screens can be better understood and assimilated by students. In addition further discussions about astronomy and its relation with polarization 
can be performed due to the use of the IRIS software and photometry analysis.

\section{Acknowledgements}

The authors would like to thank Dr. Marcelo F. Santos and Dr. Carlos Henrique Monken for their support. The authors also acknowledge support from Conselho Nacional de Desenvolvimento Científico e Tecnológico.

\section{References}

[1] E. Malus, J. Ec. Poly. 7, 1 44, (1808).

[2] E. Faggiano, in: Proceedings of CERME6, Technologies and Resources in Mathematical Education, Lyon, p. 1310-1319 (2009).

[3] S.M. Coelho, Contribution a Letude Didactique du Mesurage en Physique dans Lenseignement Secondaire. Description et Analyse de l Activit Intellectuelle et Pratique des lves et des Enseignants, U.F. de Didactique des Disciplines, Universit de Paris VII, Paris, 256 p. (1993).

[4] See supplementary material at ww. pasco.com/ prodCatalog/EX/EX-9917polarization-oflight-experimentscienceworks/\#overviewTab for an explanatory experiment.

[5] IRIS homepage http://www.astrosurf.com/ buil/index.htm accessed 2/2016.

[6] H Fakhruddin, Phys. Teach. 46, 229-231 (2008).

[7] T.M Ciferno, R.J. Ondris-Crawford and G.P. Crawford, Phys. Teach. 33, 104 (1995).

[8] S.C Gladden, Am. J. Phys. 18, 395 (1950).

[9] M. Bass, Handbook of Optics, Third Edition Volume II: Design, Fabrication and Testing, Sources and Detectors, Radiometry and Photometry (McGrawHill, New York, 2009).

[10] Bureal International des Poids et Mesures, The photometric base unit the candela, http:// www1.bipm.org/utils/en/pdf/SIApp2cden.pdf, accessed 2/2016.

[11] H. Romanishin, An Introduction to Astronomical Photometry Using CCDs (University of Oklahoma, Norman, 2006), p. 9-175.

[12] R. Eisberg, R. Resnick e J. Walker, Fundamentos de Física (LTC, Rio de Janeiro, 2000), 6th ed, v. 4.

[13] R.W.G. Hunt, The Reproduction of Colour (John Wiley \& Sons, New York, 2004), 6ht ed.

[14] M. Bolte, Modern Observational Techniques: Signalto-Noise in Optical Astronomy Class Lecture, University of California, Santa Cruz(2008).

[15] D. Halliday, R. Resnick and J. Walker, Fundamentals of Physics (Wiley, New York, 2000), 6th ed.

[16] M. Bass, Handbook of Optics, Third Edition Volume I: Geometrical and Physical Optics, Polarized Light,
Components and Instruments (McGraw-Hill, New York, 2009).

[17] B. Kahr and K. Claborn, ChemPhysChem 9, 4358 (2008)

[18] S. Chandrasekhar, Liquid Crystals (Cambridge University Press, Cambridge, 1992).

[19] L. Vertchenko and L. Vertchenko, Abakós 3, 108 (2015).

[20] http://courses.coe.uh.edu/brobin/ resources/digitalphotography/CameraFunctions.pdf.

[21] Origin homepage, http://www.originlab.com/ accessed 2/2016.

[22] Microsoft Paint homepage http://windows microsoft.com/enus/windows7/products/ features/paint, accessed 2/2016. 